\title{
Mesenchymal Stem Cell Adhesion on Metal and Polymeric Surfaces Modified by Oxygen Cluster Ion Irradiation
}

\author{
Gikan H. Takaoka ${ }^{1}$, Ryou Tsujinaka ${ }^{1}$, Mitsuaki Takeuchi ${ }^{1}$, Hiromichi Ryuto ${ }^{1}$ \\ and Hiroshi Tsuji ${ }^{2}$ \\ ${ }^{1}$ Photonics and Electronics Science and Engineering Center, Kyoto University, \\ ${ }^{2}$ Department of Electronic Science and Engineering, Kyoto University, \\ Katsura, Nishikyo-ku, Kyoto 615-8510, Japan \\ * Corresponding author: Fax: 81-75-383-2343, e-mail: gtakaoka@kuee.kyoto-u.ac.jp
}

Polyethylene (PE) and Ti surfaces were irradiated with oxygen cluster ions. For PE surfaces, the sputtered depth increased with an increase in acceleration voltage, and the sputtering yield was approximately 243 molecules per ion at an acceleration voltage of $9 \mathrm{kV}$. Atomic force microscopy showed that surface roughness increased with an increase in acceleration voltage. The contact angle was between $70^{\circ}$ and $80^{\circ}$, which was smaller than that for the unirradiated surface. Adhesion of mesenchymal stem cells (MSCs) could be obtained by adjusting acceleration voltage, although cell adhesion was not observed on the unirradiated surfaces. For Ti surfaces, the surface roughness did not change with cluster ion irradiation. However, the contact angle decreased with an increase in acceleration voltage, and it was $46^{\circ}$ at an acceleration voltage of $9 \mathrm{kV}$. Furthermore, after ultraviolet light irradiation for $30 \mathrm{~min}$, the contact angle decreased to $28^{\circ}$. With regard to cell adhesion, the number of MSCs attached to the Ti surface increased after oxygen cluster ion irradiation.

Key words: Oxygen cluster, Ion beam, Stem cell, Cell adhesion, Titanium, Polyethylene

\section{INTRODUCTION}

Mesenchymal stem cells (MSCs) and the extracellular matrix (ECM) constituting tissues have been studied from the perspective of biological materials [1]. For example, MSCs, which have self-renewal and differentiation potential, have been shown to differentiate into many cell lines such as bone, tendon, ligament, muscle cells, and dermal cells $[2,3]$. Cells adhere to each other and to ECM through intracellular attachment proteins to form cell adhesion patterns. Adhesive proteins contained in the ECM have an important role in cell adhesion. In general, proteins consist of both hydrophobic and hydrophilic bonds, and they are randomly adsorbed on surfaces. Therefore, both ultra-hydrophilic surfaces and ultra-hydrophobic surfaces are not suitable for promoting protein adsorptions. With regard to the wettability of material surfaces, the contact angle of a water droplet on a surface is an appropriate parameter reflecting wettability, and a suitable contact angle for cell adhesion lies between $40^{\circ}$ and $80^{\circ}[4,5]$.

For the enhancement of cell adhesion or for the improvement of biocompatibility of metal and polymeric materials, various kinds of surface modification methods such as plasma processing, ion implantation, and surface coating are available [6-9]. Cluster ion beams have several advantages for material surface modification [10-13]. For example, high-density irradiation enhances the surface temperature of the impact region, which enhances the chemical reactions occurring on the surface. Therefore, oxygen cluster ion beams can enhance surface oxidation even at room temperature.

In recent years, oxide materials have attracted much interest, and they are applied to various fields in terms of oxide electronics such as oxide superconductor electronics, optoelectronics, photocatalysis, biophotonics, and biomimetics [14-18]. The surface modification technique based on oxygen cluster ion beams has a high potential for the surface oxidation of various materials with low irradiation damage. For example, for metal and semiconductor materials, surface oxidation is a useful method for passivation and for enhancing the chemical stability of surfaces. In photocatalysis, the functionality of surfaces is enhanced [19,20]. In addition, oxidation of polymeric surfaces enhances the wettability of the surfaces [21], which results in improved cell adhesion to the surfaces.

In this study, oxygen cluster ion beams were used to irradiate $\mathrm{Ti}$ and polyethylene (PE) substrates. PE has attracted much interest as a useful polymer for industrial and medical applications [22,23], because it is a light, stable, and flexible material. Cell adhesion on PE surfaces has an important role in improving biocompatibility. In addition, $\mathrm{Ti}$ is used as an implant material in the living body [24], because it exhibits good biocompatibility and has excellent bonding ability for bone and tooth tissue. In general, foreign body response is invoked as the initial stage of the body's natural defense system. The acute inflammation phase can last from hours to days, depending on the surgical procedure [8]. To improve the biocompatibility of implant materials, one approach for minimizing the acute inflammation phase is surface modification, resulting in enhanced cell adhesion to the implant surface. Furthermore, $\mathrm{Ti}$ used as artificial bone has higher fracture toughness and stiffness than bone, which prevents the need for stress transfer from the adjacent bone. This results in bone resorption around the implant, leading to implant loosening. Cell adhesion is of considerable importance to improve bonding ability on 
the implant surface. Thus, cell adhesion on Ti surfaces plays a central role in engineering any kind of biological tissue, and it offers biological stability through tissue regeneration. Therefore, in the present study, MSCs were cultured in vitro, and cell adhesion to irradiated $\mathrm{Ti}$ and PE surfaces was investigated.

\section{EXPERIMENT}

The details of the experimental apparatus are described elsewhere [25]. High-pressure oxygen gas was ejected through a glass nozzle into a vacuum region. When the inlet pressure was more than $0.3 \mathrm{MPa}$, neutral oxygen cluster beams were produced by adiabatic expansion. The average size (peak size) of oxygen clusters was approximately 1800 molecules per cluster at an inlet pressure of $0.45 \mathrm{MPa}$. The cluster beams formed were ionized by electron bombardment. The ionization voltage $\left(V_{\mathrm{e}}\right)$ was $200 \mathrm{~V}$, and the electron current for ionization $\left(I_{\mathrm{e}}\right)$ was $200 \mathrm{~mA}$. Oxygen cluster ion beams were accelerated towards substrates and irradiated on them. The acceleration voltage $\left(V_{\mathrm{a}}\right)$ was adjusted between $1 \mathrm{kV}$ and $10 \mathrm{kV}$. The fluence was maintained at a constant value of $1.0 \times 10^{15}$ ions $/ \mathrm{cm}^{2}$. The substrates used were Ti and PE substrates, and the substrate temperature was room temperature. The surface roughness of irradiated substrates was measured by atomic force microscopy (AFM). The composition and chemical binding state of irradiated substrates were measured by X-ray photoelectron spectroscopy (XPS).

With regard to cell culture, the substrates irradiated by oxygen cluster ions were sterilized by ultra violet (UV) light in a clean bench for $12 \mathrm{~h}$ and were rinsed by sterilized de-ionized water. They were set in a polystyrene dish and fixed by using $0.33 \%$ agarose. Rat MSCs were cultured in vitro for 7 days on the Ti and PE substrates. In the case of PE substrates, a micro-pattern mask was used for cell adhesion patterning, and it consisted of many slit apertures $50 \mu \mathrm{m}$ wide, $5 \mathrm{~mm}$ long and $150 \mu \mathrm{m}$ apart.

The cell adhesion properties on the modified substrates were observed by fluorescent microscopy (BX50, Olympus). Cell shape was identified by labeling of actin filaments (F-actin) with Texas Red fluorescent dye. F-actin is the thin and flexible protein fiber formed by the polymerization of globular actin molecules, and the staining of actin filaments can be used as an identifying marker to determine cell shape. Positions of adhered cells were identified by labeling cell nuclei. For confirmation of actual position of nucleus adhesion, 4'-, 6-diamidino-2-phenylindole immunofluorescent (DAPI) was applied, and blue fluorescence was assessed.

\section{RESULT AND DISCUSSION}

\subsection{PE substrate}

To improve cell adhesion, PE surfaces were oxidized by oxygen cluster ion irradiation. The sputtered depth was measured by a step profiler (Veeco Instruments: DEKTAK-3173933). Figure 1 shows the dependence of sputtered depth for PE substrates on the acceleration voltage. The sputtered depth increased with increase in acceleration voltage and was $118 \mathrm{~nm}$ at an acceleration voltage of $9 \mathrm{kV}$. Taking into account the sputtered depth and fluence, the sputtering yield was calculated by estimating the density of PE as $0.96 \mathrm{~g} / \mathrm{cm}^{3}$. The sputtering yield of PE at an acceleration voltage of $9 \mathrm{kV}$ was approximately 243 molecules per ion if the sputtered molecule was ejected from the PE surface as a monomer state. The high rate of sputtering was due to the high-density irradiation effect by oxygen cluster ion beams.

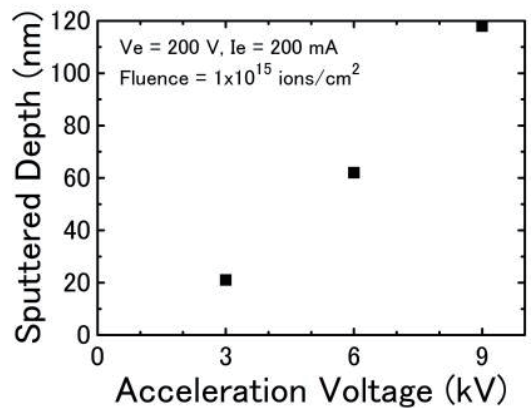

Fig.1 Dependence of sputtered depth for PE substrates on the acceleration voltage.

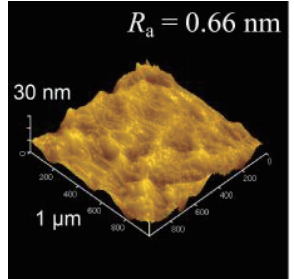

(a) Unirradiated

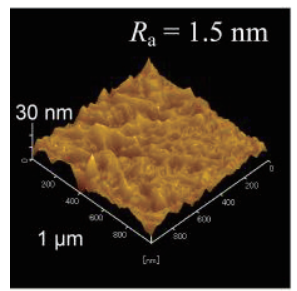

(c) $V_{\mathrm{a}}=6 \mathrm{kV}$

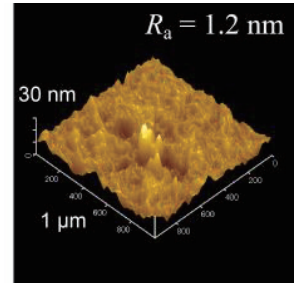

(b) $V_{\mathrm{a}}=3 \mathrm{kV}$

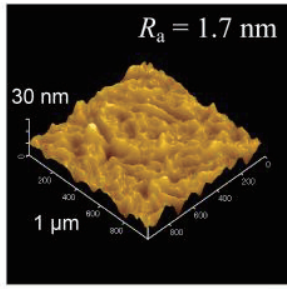

(d) $V_{\mathrm{a}}=9 \mathrm{kV}$
Fig.2 Surface morphology and roughness of PE substrates irradiated at different acceleration voltages.

Figure 2 shows the surface morphology and roughness of the PE substrates irradiated at different acceleration voltages by oxygen cluster ion beams. The surface roughness increased with an increase in acceleration voltage, and it was $1.7 \mathrm{~nm}$ at an acceleration voltage of $9 \mathrm{kV}$. The surface roughness of the unirradiated surface was $0.66 \mathrm{~nm}$. Thus, cluster ion beams have unique characteristics for PE surface treatment with high sputtering yield and smooth surface at the atomic level.

XPS measurements showed that the irradiated surfaces exhibited several $\mathrm{C} 1 \mathrm{~s}$ peaks corresponding to $\mathrm{C}-\mathrm{C}, \mathrm{C}-\mathrm{O}$, and $\mathrm{C}=\mathrm{OO}$ bonds. This indicated that the $\mathrm{PE}$ surfaces were oxidized by oxygen cluster ion irradiation. From these peaks, the oxygen component was calculated. Figure 3 shows the oxygen atom ratio for the PE surfaces irradiated at different acceleration voltages. The oxygen component decreased with an increase in acceleration voltage, and the surface oxidation was enhanced considerably at a lower acceleration voltage of $3 \mathrm{kV}$. 
To evaluate the wettability of PE surfaces, contact angles were measured for water droplets applied on the surfaces just after their removal from a vacuum chamber. Figure 4 shows the dependence of the contact angle on the acceleration voltage for the PE substrates irradiated by oxygen cluster ion beams. The contact angle prior to the oxygen cluster ion irradiation was $102^{\circ}$, and the surface exhibited hydrophobicity. On the other hand, the contact angle for the irradiated surfaces decreased, and it was between $70^{\circ}$ and $80^{\circ}$. It is generally considered that the contact angle for PE surfaces might increase with an increase in surface roughness. However, the contact angle decreased, and the hydrophilicity of the PE surfaces was enhanced. This can be attributed to the surface oxidation by oxygen cluster ion irradiation.

Figure 5 shows the fluorescent micrographs of F-actin on the PE surfaces irradiated at different acceleration voltages by oxygen cluster ion beams. The number of MSCs increased and reached the confluence state after culturing for 7 days. As shown in the figure, most of the MSCs adhered selectively and spread only on the irradiated region. At acceleration voltages higher than $3 \mathrm{kV}$, degradation of the selective adhesion property occurred, and some cells were hanging out of the irradiated region at the acceleration voltage of $9 \mathrm{kV}$. This might be due to the aggregation of cells adhered under the mask, which was caused by the enhancement of the oxidation for the unirradiated region of the PE surface.

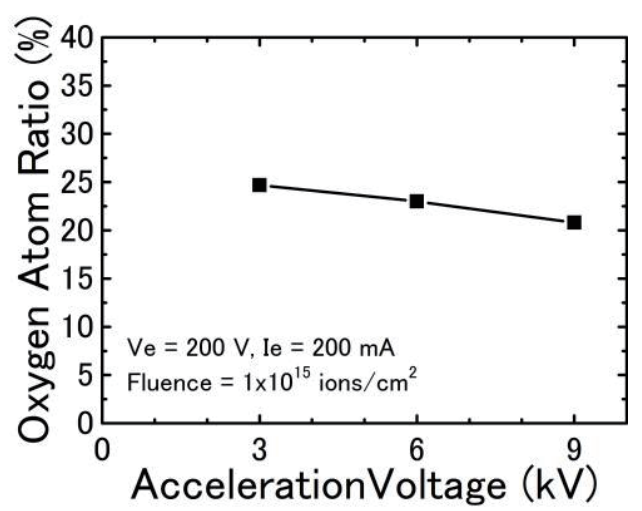

Fig.3 Oxygen atom ratio for the PE surfaces irradiated at different acceleration voltages.

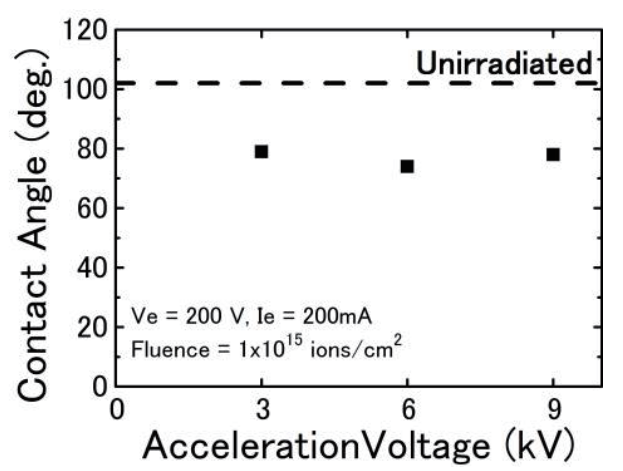

Fig.4 Dependence of contact angle on the acceleration voltage for PE substrates irradiated by the oxygen cluster ion beams.

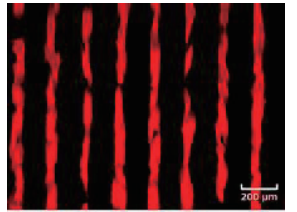

(a) $V_{\mathrm{a}}=3 \mathrm{kV}$ (F-actin)

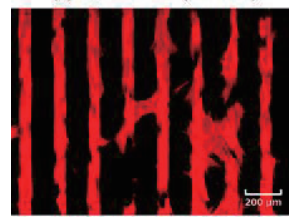

(b) $V_{\mathrm{a}}=6 \mathrm{kV}$ (F-actin)

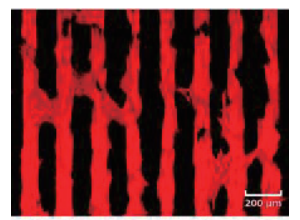

(c) $V_{\mathrm{a}}=9 \mathrm{kV}$ (F-actin)

Fig.5 Fluorescent micrographs of F-actin on the $\mathrm{PE}$ surfaces irradiated at different acceleration voltages.

\subsection{Ti substrate}

Surface modification of Ti surfaces was performed by oxygen cluster ion irradiation, and titanium oxide surfaces were formed. Titanium oxide has attracted much attention from the view point of photocatalysis. Its wettability can be changed by UV irradiation, and the contact angle can be adjusted to the optimum value for cell adhesion. Figure 6 shows UV irradiation-time dependence of the contact angles for $\mathrm{Ti}$ surfaces irradiated at different acceleration voltages by oxygen cluster ion beams. The wavelength of the UV light used was $360 \mathrm{~nm}$. As shown in the figure, the contact angle for $\mathrm{Ti}$ surface prior to the oxygen cluster ion irradiation was $84^{\circ}$, and it decreased to $68^{\circ}$ after UV irradiation. In contrast, for the titanium oxide surfaces prepared at the acceleration voltage of $9 \mathrm{kV}$, the contact angle changed from $46^{\circ}$ to $28^{\circ}$ after UV irradiation. As a result, the wettability of the Ti surfaces was enhanced by the oxygen cluster ion irradiation, and the contact angle was controlled by UV irradiation time.

The surface state of irradiated Ti substrates was measured by an optical microscope. Figure 7 shows the surface morphology and the roughness of the $\mathrm{Ti}$ substrates (a) before and (b) after oxygen cluster ion irradiation. The acceleration voltage was $9 \mathrm{kV}$. As shown in the figure, the surface morphology did not change by the irradiation. In addition, the surface roughness (Ra) measured by DEKTAK was $0.17 \mu \mathrm{m}$, and it was similar to that for the unirradiated surface. Furthermore, the surface roughness measured by AFM was $0.14 \mathrm{~nm}$, and it was almost the same as that for the unirradiated surface. It should be noted that surface roughness, which influences cell adhesion, should be of the same order as that of cell length, which is in the micrometer range.

In vitro cell adhesion on the UV irradiated $\mathrm{Ti}$ and titanium oxide surfaces was investigated by fluorescent 
microscopy. The titanium oxide surfaces were prepared at an acceleration voltage of $9 \mathrm{kV}$ by oxygen cluster ion beams. Figure 8 shows the fluorescent micrographs of MSCs on the $\mathrm{Ti}$ and titanium oxide surfaces after culturing for $48 \mathrm{~h}$. As shown in panels (a) and (b), F-actin and nuclei were observed for Ti surfaces, and MSC adhesion at the confluence state was obtained. The number of nuclei was $1.46 \times 10^{4}$ cells $/ \mathrm{cm}^{2}$. However, as shown in panels (c) and (d), the number of the nuclei increased to $2.63 \times 10^{4}$ cells $/ \mathrm{cm}^{2}$. Proteins contained in MSCs arrive at the surface site through convective transport in the tissue fluid. This is often observed in the bulk fluid located too far away for any interacting forces have a significant effect. This region is referred to as the diffusive transport region, and it is typically $10 \mu \mathrm{m}$ long [8]. Proteins that are carried to the site of Ti and titanium oxide surfaces by convective transport diffuse through this diffusion-controlled region before reaching the surface. At this close proximity region $(2-5 \mathrm{~nm})$, short-range forces such as hydrogen bonding and electrostatic forces are dominant, and the interaction between the surface and the protein determine the extent of MSC adhesion. Hydroxyl radicals are produced from titanium oxide surfaces irradiated by UV-light, and they have an important role in decreasing the contact angle. In addition, cell adhesion through protein absorption is enhanced owing to hydroxyl radicals, which are effective for forming hydrogen bonds.

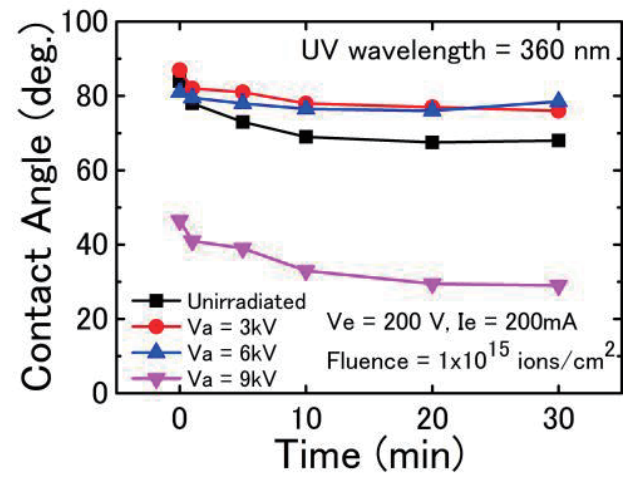

Fig.6 UV-irradiation-time dependence of contact angles for $\mathrm{Ti}$ surfaces irradiated at different acceleration voltages.

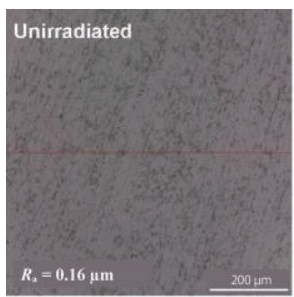

(a) Prior to ion irradiation

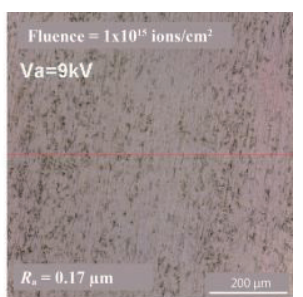

(b) After ion irradiation
Fig.7 Surface morphology and roughness of the Ti substrates (a) before and (b) after the oxygen cluster ion irradiation. The acceleration voltage was $9 \mathrm{kV}$.

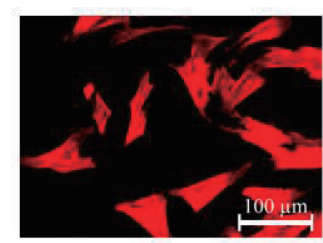

(a) Unirradiated (F-actin)

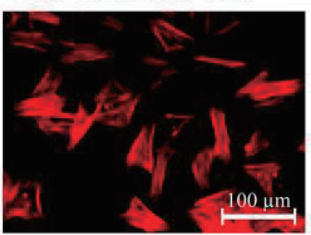

(c) Ion Irradiation (F-actin)

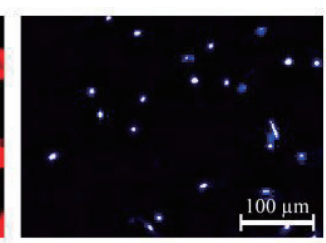

(b) Unirradiated (Nucleus)

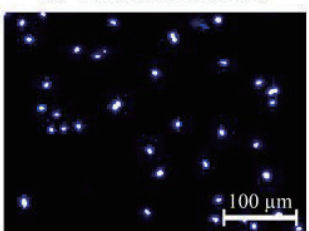

(d) Ion Irradiation (Nucleus)
Fig.8 Fluorescent micrographs of MSCs on the Ti and titan oxide surfaces after cultured for 48 hours

Figure 9 shows the number of MSCs adhered to the $\mathrm{Ti}$ and titanium oxide surfaces at different culture times. The Ti and titanium oxide surfaces were not irradiated by UV light. As shown in the figure, the number of MSCs adhered to the titanium oxide surface was larger than that on the Ti surface. The oxide sites have a central role in anchoring cell adhesion, which results in an increase in cell numbers. In addition, adhesion strength is expected to be improved, resulting in the formation of surface stable enough for biomedical applications. At 12 and $24 \mathrm{~h}$, the number of cells decreased. This was accidentally caused by a change in the fluid condition, although exact details of the cause are under investigation.

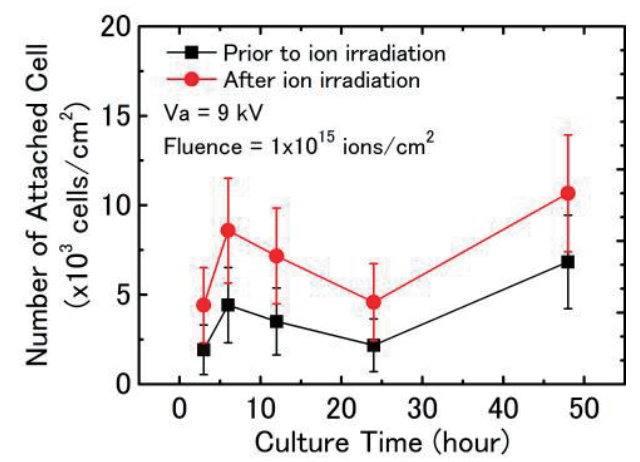

Fig.9 The number of MSCs adhered on the Ti and titan oxide surfaces at different culture times.

\section{CONCLUSIONS}

Oxygen cluster ion beams were irradiated on $\mathrm{Ti}$ and PE surfaces. The acceleration voltage was adjusted to be between $1 \mathrm{kV}$ and $10 \mathrm{kV}$, and the fluence was constant at $1.0 \times 10^{15}$ ions $/ \mathrm{cm}^{2}$. For PE surfaces, the sputtered depth increased with an increase in acceleration voltage, and sputtering yield was approximately 243 molecules per ion at an acceleration voltage of $9 \mathrm{kV}$. The surface 
roughness increased with an increase in acceleration voltage, although it was less than $1.7 \mathrm{~nm}$. Cell adhesion could be achieved by oxygen cluster ion irradiation, although cell adhesion was not observed on unirradiated surfaces. The enhancement of cell adhesion was ascribed to the oxidation of PE surfaces by the oxygen cluster ion irradiation. Furthermore, MSC adhesion patterning was demonstrated on the PE surfaces, and the optimum acceleration voltage for the patterning was $3 \mathrm{kV}$.

For $\mathrm{Ti}$ surfaces irradiated by oxygen cluster ion beams, the surface roughness was similar to that before cluster ion irradiation. However, the contact angle decreased with an increase in the acceleration voltage, and it was $46^{\circ}$ at an acceleration voltage of $9 \mathrm{kV}$. Furthermore, after UV light irradiation for $30 \mathrm{~min}$, the contact angle decreased to $28^{\circ}$. The decrease in contact angle was ascribed to the photocatalytic ability of the titanium oxide surfaces formed by oxygen cluster ion irradiation. With regard to cell adhesion, the number of MSCs attached to the titanium oxide surfaces was larger than that on $\mathrm{Ti}$ surfaces prior to the cluster ion irradiation.

\section{ACKNOWLEDGEMENT}

The authors would like to acknowledge Nissin Ion Equipment Co. Ltd for the supply of Ti substrates.

\section{REFERENCES}

[1] C. K. Kuo and M. L. Smith, MRS Bull. 39 (2014) 19.

[2] R.L. Gardner, J. Anat. 200 (2002) 277

[3] S. Bobis, D. Jarocha and M. Majka, Folia Histochemica Et Cytobiologica, 44 (2006) 215.

[4] N. G. Maroudas, Inter. Rev. Cytology 53 (1978) 65.

[5] F. Grinnel, Nature 254 (1975) 695.

[6] Y. Suzuki, M. Kusakabe, K. Kusakabe, H. Akiba and M. Iwaki, Nucl. Instrum. Methods B59-60 (1991) 698.

[7] H. Tsuji, H. Satoh, S. Ikeda, S. Ikemura, Y. Gotoh and J. Ishikawa, Nucl. Instrum. Methods B148 (1999) 1136.

[8] P. Lin, C-W. Lin, R. Mansour and F. Gu, Biosensors \& Bioelectronics 47 (2013) 451.

[9] T. Cheng, Y. Chen and X. Nie, Thin Solid Films, 549 (2013) 123.

[10] I.Yamada, Eur. Phys. J. D. 9, (1999) 55.

[11] G. H. Takaoka, H. Ryuto and M. Takeuchi, J. Mater. Res. 27 (2012) 806

[12] G. H. Takaoka, H. Ryuto, M. Takeuchi and H. Kobayashi, Nucl. Instrum. Methods B326 (2014) 190.

[13] G. H. Takaoka, H. Ryuto, M. Takeuchi and R. Tsujinaka, Proc. 20th Int. Conf. on Ion Implant. Technol. (AIP Conf. Proc. 2014) p.307.

[14] K. Sakai, M.M. Saari, T. Kiwa, A. Tsukamoto, S Adachi, K. Tanabe, A. Kandori, and K. Tsukada, Supercond. Sci. Technol. 25 (2012) 045005.

[15] M.A. Hernandez-Rodriguez, M.H. Imanieh, L.L. Martin, and I.R. Martin, Solar Energy Mater. \& Solar Cells, 116 (2013) 171.

[16] K. Nomura, H. Ohta, A. Takagi, T. Kamiya, M. Hirano, and H. Hosono, Nature, 432 (2004) 488.

[17] M. Grundmann, H. Frenzel, A. Lajin, M. Lorenz, F. Achein and H.V. Wenckstern, Phys. Status Solidi A, 207 (2010) 1437.
[18] S.D. Ha and S. Ramanathan, J. Appl. Phys. 110 (2011) 071101.

[19] G.H. Takaoka, M. Kawashita, K. Omoto and T. Terada, Nucl. Instrum. Methods B232 (2005) 200.

[20] G. H. Takaoka, T. Nose and M. Kawashita, Vacuum 83 (2009) 679.

[21] G. H. Takaoka, M. Kawashita, H. Shimatani and R. Araki, Surf. Coat. Technol. 201 (2007) 8242.

[22] M. Kawashita, H. Shimatani, S. Itoh, R. Araki and G. H. Takaoka, J. Ceramic Soc. Jpn. 114 (2006) 77.

[23] M. Kawashita, S. Itoh, K. Miyamoto, R. Araki and G.H. Takaoka, Key Eng. Mater. 330-332 (2007) 111.

[24] T. Hayakawa, M. Kawashita, G. H. Takaoka and T. Miyazaki, J. Mater. Res. 23 (2008) 3176.

[25] N. Toyoda, N. Hagiwara, J. Matsuo and I. Yamada, Nucl. Instrum. Methods B148 (1999) 639.

(Received August 21, 2014; Accepted November 25, 2014) 